Cahiers $d u$ MONDE RUSSE

\section{Cahiers du monde russe}

Russie - Empire russe - Union soviétique et États indépendants

$51 / 4 \mid 2010$

Sciences humaines et sociales en Russie à l'Âge d'argent

\title{
Tanja Penter, Kohle für Stalin und Hitler
}

\section{Nathalie Moine}

\section{(2) OpenEdition}

Journals

Édition électronique

URL : https://journals.openedition.org/monderusse/7397

DOI : 10.4000/monderusse.7397

ISSN : $1777-5388$

Éditeur

Éditions de l'EHESS

Édition imprimée

Date de publication : 25 novembre 2010

Pagination : 726-731

ISBN : 978-2-7132-2316-7

ISSN : $1252-6576$

Référence électronique

Nathalie Moine, "Tanja Penter, Kohle für Stalin und Hitler », Cahiers du monde russe [En ligne], 51/4 I 2010, mis en ligne le 09 décembre 2011, consulté le 03 septembre 2022. URL : http://

journals.openedition.org/monderusse/7397; DOI : https://doi.org/10.4000/monderusse.7397

Ce document a été généré automatiquement le 3 septembre 2022

Tous droits réservés 


\title{
Tanja Penter, Kohle für Stalin und Hitler
}

\author{
Nathalie Moine
}

\section{RÉFÉRENCE}

Tanja PENTER, Kohle für Stalin und Hitler. Arbeiten und Leben im Donbass, 1929 bis

1953. Essen : Klartext Verlag, 2010, 467 p.

1 Le projet de Tanja Penter était ambitieux et se révèle réussi : retracer l'histoire des habitants du bassin minier du Donbass de la fin des années 1920 à 1953, soit sur l'ensemble de la période stalinienne, en y incluant pleinement la période d'occupation nazie, ce que reflète le titre, « Du charbon pour Stalin et Hitler ». Cette étude s'inscrit résolument dans une perspective d'histoire sociale, qui s'exprime dans le sous-titre: "Travail et vie [quotidienne] dans le Donbass, 1929-1953». Ses principaux axes reprennent des thématiques largement traitées dans l'historiographie des vingt dernières années en ce qui concerne le «premier stalinisme» - industrialisation à marche forcée, grands chantiers -, mais nettement moins abordées pour ce qui est du second stalinisme reconstruction -, voire inexistantes quand on considère la période intermédiaire, à savoir l'histoire des territoires soviétiques occupés. Ainsi, les questions du recrutement de la main-d'œuvre et le recours au travail forcé, les conditions de vie (et singulièrement le ravitaillement), enfin la construction d'une identité collective à travers une consommation culturelle marquée par une propagande intensive structurent l'ouvrage et donnent leur unité aux trois grandes parties chronologiques qui le composent. D'emblée est donc posée la question de la comparaison entre premier et second stalinisme, mais aussi entre pratiques staliniennes et pratiques nazies en territoire occupé soviétique.

2 Si le prologue nous rappelle que le charbon du Donbass a été découvert sous Pierre le Grand et que la région connaît un premier essor industriel à partir des années 1870, c'est bien avec la nouvelle naissance du bassin houiller que s'ouvre véritablement le récit, 
puisque le Donbass constitue une pièce maîtresse de l'industrialisation forcée décidée par Stalin, la production de charbon doublant en dix ans. Malgré l'afflux de population, le manque chronique de main-d'œuvre est un problème important qui, pourtant, n'est pas résolu par le recours au travail forcé des paysans dékoulakisés, contrairement à d'autres grands chantiers comme le Kuzbass ou Magnitogorsk. L'autre problème récurrent est celui de l'encadrement. Même si l'organisation de procès dès le début des années 1920 montre que le phénomène n'est pas tout à fait nouveau, ingénieurs et autres cadres font l'objet de violentes attaques au cours du premier stalinisme, depuis les procès publics de la fin des années 1920, notamment celui, très célèbre, de Šahty, jusqu'aux procès et arrestations de la Grande Terreur, en passant par la forte hostilité développée à leur égard à l'occasion des campagnes stakhanovistes.

Fondé sur une histoire du travail, cet ouvrage constitue aussi l'histoire d'une région, et ces deux aspects se trouvent imbriqués dans la question de la famine de 1932-1933. Région ukrainienne, le Donbass est aussi une région privilégiée par le Kremlin. De fait, la crise alimentaire se fait sentir, notamment dans son contexte régional avec l'afflux de nombreux bežency [réfugiés] dans les villes et les bourgs miniers. Pourtant, si la population urbaine du Donbass est mieux servie que les autres - et, en son sein, les travailleurs de l'industrie, selon des critères hiérarchiques désormais bien connus -, c'est l'ensemble de la population de la région qui souffre un peu moins, puisque les ruraux du Donbass connaissent des taux de mortalité moins élevés que le reste de l'Ukraine. Ainsi, paradoxalement, les habitants des bourgs miniers, qui sont les premières victimes du boom industriel $\mathrm{du}$ fait de conditions de vie très dégradées (absence d'approvisionnement en eau, de réseaux d'égouts, de chauffage central), sont aussi les mieux placés pour échapper à la mort par la famine, du fait du réseau de cantines et de magasins, mêmes hiérarchisés, qui assurent un approvisonnement minimal. En revanche, les formes d'auto-alimentation liées à la possession d'un lopin ou de petit bétail, traditionnelles chez le mineur au mode de vie hybride entre ville et campagne, connaissent une diminution drastique dans la première moitié des années 1930, sans doute du fait de l'arrivée massive d'une nouvelle main-d'œuvre (cf. tableau p. 89).

Si la crise dans ses aspects les plus aigus est surmontée, les conditions misérables en font fuir plus d'un. Parmi les désenchantés, le groupe atypique des ouvriers étrangers, aux conditions pourtant privilégiées. La rencontre entre deux mondes est également illustrée par un phénomène mal connu dans l'historiographie et pourtant bien plus massif : l'envoi de milliers d'habitants en provenance des territoires annexés de 1939-1940 - chômeurs, souvent juifs, de Galicie, paysans ou ouvriers non qualifiés de Bukovine ou de Bessarabie -, qui se solde par de nombreux retours, le plus souvent clandestins, pour fuir les conditions de vie et de travail du Donbass.

5 Tanja Penter est également attentive à restituer l'identité officielle qui se construit au cours des années 1930, même s'il est difficile d'évaluer à quel point elle est intégrée par les habitants. La production culturelle officielle de l'époque, des ensembles folkloriques à la presse locale, contribue à fabriquer une identité plus régionale qu'ukrainienne, dans laquelle la figure du mineur acquiert une stature centrale et devient, grâce notamment au cinéma, un héros du socialisme dans toute l'URSS.

6 Faut-il dès lors voir l'occupation comme une rupture? La construction de l'ouvrage et son questionnement insistent au contraire sur une certaine continuité. En même temps, l'auteur, par la précision et la diversité des aspects étudiés pour cette période, établit fermement les différences. Pour les nazis, le Donbass acquiert une importance centrale 
dans le fonctionnement économique de l'ensemble des territoires occupés soviétiques. Un effort particulier est donc fait pour reconstruire l'appareil industriel détruit par les Soviétiques au moment de leur retraite. Comme avant la guerre, la question de la maind'œuvre est cruciale et conduit les autorités nazies à ne pas suivre certaines décisions, prises à l'encontre des populations soviétiques par les planificateurs de l'invasion : celles qui concernent le recrutement, vite remplacé par la déportation forcée, pour travailler dans le Reich, et l'absence de ravitaillement des villes. L'objectif étant de s'assurer un contingent suffisant pour reconstruire les mines et produire le charbon, les autorités vont tenter de maintenir sur place la main-d'œuvre et de lui offrir de quoi survivre. Tanja Penter offre ainsi une intéressante discussion sur la notion de travail forcé. Alors que Paul Pleiger, principal responsable économique à l'Est, est accusé après-guerre des pratiques esclavagistes utilisées dans les établissements du BHO (Berg- und Hüttenwerksgesellschaft mbH Ost, la Société des mines et aciéries de l'Est) en Union soviétique, les victimes qui ont été forcées de travailler dans les mines en territoire soviétique occupé tout en restant chez elles ne bénéficieront pas du programme de compensation lancé par les Allemands après 1991. Pourtant, les normes de rationnement en vigueur dans les mines du Donbass étaient encore plus basses que pour les Ostarbeiter soviétiques déportés dans le Reich. Plus bas encore dans l'échelle du travail forcé, on trouve les prisonniers de guerre soviétiques, qui se révèlent pour moitié inaptes au travail et, pour le reste, sont employés à la surface car ils sont trop faibles pour travailler au fond ; leurs effectifs fondent rapidement du fait de leur très forte mortalité. Dans cet apport essentiel de Tanja Penter à l'histoire de la mise au travail en territoire occupé soviétique, le comportement de l'encadrement constitue également un aspect passionnant. Beaucoup des anciens cadres reprennent du service dans la mine, aident les Allemands à reconstruire l'appareil de production mais aussi à retrouver les ouvriers qualifiés. La question des formes de collaboration et de réemploi des structures et de l'expertise soviétique est posée plus largement dans l'étude des administrations locales laissées aux mains des autochtones bien que sous un contrôle serré des Allemands.

Les exécutions de masse sont le deuxième grand aspect de l'occupation, qui rapproche et distingue tout à la fois expérience stalinienne et nazie. L'échelle des massacres distingue clairement l'occupation y compris de la phase sanglante de la Grande Terreur de 1937-1938, même si le nombre de victimes retrouvées dans les charniers à la Libération est très certainement surévalué par les Soviétiques. La part respective des juifs, des civils non juifs et des prisonniers de guerre soviétiques reste également sujette à caution.

traite des Allemands du Donbass constitue aussi un moment mal connu de l'histoire de l'occupation. Les destructions sont à nouveau massives. En ce qui concerne l'évacuation de la population, elle n'a pas l'ampleur planifiée par les autorités allemandes, mais concerne tout de même plusieurs centaines de milliers de personnes.

Comme dans les années 1930, misère et violence extrêmes contrastent avec l'image de la région véhiculée par la propagande nazie. La vie culturelle s'est en réalité raréfiée pour les habitants du Donbass pendant l'occupation, du fait d'un nombre plus réduit de lieux de spectacles (théâtre, cinéma). Il est aussi intéressant de noter que des films soviétiques de l'entre-deux-guerres, considérés par les autorités d'occupation comme " apolitiques ", tels Volga Volga ou Veselye rebjata ${ }^{1}$, continuent d'être projetés pendant la guerre. Une presse d'occupation se développe, en langue russe et ukrainienne, mais sous contrôle direct des services de la propagande allemande qui font appel à des journalistes locaux dont certains ont travaillé pour la presse soviétique d'avant-guerre. Tanja Penter 
souligne les correspondances entre les thématiques développées par la presse collaborationniste et soviétique : caractère central du travail, émulation entre mineurs, avenir radieux promis au bassin minier du Donbass, culte de la personnalité ; l'intérêt de cette étude réside aussi, bien entendu, dans ce qui en diffère radicalement : propagande antisémite et aussi exposition de la violence stalinienne qui trouve pour la première fois une expression publique dans l'espace ukrainien - famine de 1932-1933, Terreur, camps du Goulag, ou encore destruction de la culture ukrainienne par les bolcheviks, alors que la renaissance de la culture ukrainienne, permettant le retour de l'Ukraine dans l'Europe, est exaltée.

10 S'il est difficile de mesurer l'impact de la propagande sur les populations, Tanja Penter pose cependant la question de la loyauté des habitants du Donbass vis-à-vis des nouveaux et des anciens maîtres. Des rapports allemands notent des manifestations de sympathie à l'arrivée de la Wehrmacht, des rapports soviétiques se plaignent de l'hostilité des ruraux face au retour de l'Armée rouge dans une région libérée du Donbass au début de 1943, mais rien ne permet d'évaluer véritablement l'état d'esprit de l'ensemble de la société. Si la résistance armée n'est pas massive dans cette partie de l'Ukraine, la part non négligeable de la population au service des Allemands est-elle un indice d'adhésion? Ou une simple question de survie? De fait, les différentes formes d'épuration qui marquent le retour des Soviétiques dans les territoires libérés constituent un apport important de l'ouvrage, Tanja Penter offrant une analyse approfondie des nombreux procès contre les collaborateurs locaux auxquels elle a eu accès, à la suite du travail pionnier de Kuromiya Hiroaki ${ }^{2}$.

11 L'intérêt n'est pas moindre dans ce qui relève davantage d'une histoire économique et sociale de la sortie de guerre. La reconstruction du Donbass est une priorité absolue pour le pouvoir soviétique. Contrairement aux années 1930, mais dans la continuité de la guerre, le recours à la main-d'œuvre forcée y est massif et Tanja Penter offre une étude très fine des différents statuts de travailleurs. Dans les faits, le rang le plus bas dans l'échelle des travailleurs forcés de la sortie de guerre est occupé par les Allemands ethniques raflés très majoritairement en Roumanie, mais aussi dans d'autres pays d'Europe du Sud et de l'Est à l'arrivée de l'Armée rouge. Viennent ensuite les prisonniers de guerre étrangers, essentiellement des Allemands. Le troisième grand contingent de travailleurs forcés est constitué par des citoyens soviétiques: les rapatriés contraints après les procédures de filtration à participer à la reconstruction du Donbass plutôt qu'à rentrer chez eux ${ }^{3}$, et enfin des prisonniers du Goulag, plutôt occupés à la reconstruction des usines. Tanja Penter examine aussi le statut ambigu des travailleurs libres soumis à un recrutement forcé, notamment les jeunes apprentis dont Donald Filtzer avait déjà retracé les terribles conditions de vie et de travail ${ }^{4}$. De façon générale, la législation sur le travail, héritée de la guerre et encore en vigueur, fait des ouvriers et mineurs du Donbass des déserteurs s'ils décident de quitter leur poste. Tanja Penter a beau montrer que la mise en œuvre de cette législation draconienne se heurte à des difficultés d'application (impossibilité de localiser le déserteur, solidarité tacite de la direction de l'entreprise) et que beaucoup y échappent, le turnover atteignant des sommets en 1946-1947, les nombreux procès publics sur ce thème contribuent cependant à l'atmosphère répressive de l'époque.

12 Si tout le monde est plus ou moins rivé à son poste de travail, la distinction entre libres et non libres n'est pas aisée non plus à l'examen de leurs conditions concrètes d'existence. En ce qui concerne l'alimentation, les rations effectivement servies ne sont 
pas forcément plus élevées chez les libres, surtout si on les compare à celles des prisonniers de guerre. L'accès à un potager peut constituer une différence essentielle (dont ne bénéficient généralement pas les jeunes apprentis).

13 Confusion des statuts et misère générale expliquent pourquoi la famine de 1946-1947 frappe les habitants du Donbass différemment de celle de 1932-1933. Notons que la population urbaine du Donbass est, cette fois, aussi durement touchée que les ruraux de la région. L'accès aux cantines se raréfie au point de ne plus concerner que $10 \%$ à peine des ouvriers dans de nombreuses mines.

Le Donbass reste un objet de propagande essentiel, mais sa représentation se révèle problématique : pour avoir montré des maisons délabrées et évoqué la collaboration avec les Allemands, la deuxième partie du film Bol'šaja žizn' [La grande vie] (1946) de Leonid Lukov, dont l'action se déroule à la libération du Donbass, devra attendre le Dégel pour être projetée sur les écrans soviétiques. Pour finir, Leonid Lukov signera avec Šahtery Donbassa [Les mineurs du Donbass] (1950), un film dont les véritables héros sont davantage des machines que des hommes.

Il n'est pas possible de rendre compte de tous les apports d'une somme qui se distingue à la fois par sa richesse et sa cohérence. Une de ses moindres qualités n'est pas la discussion sur les données chiffrées. Cependant, un prolongement possible de cette mise en perspective du stalinisme serait de tenter de quantifier pour l'ensemble de la période un certain nombre de phénomènes, tels que les taux de mortalité ruraux et urbains, notamment dans les mois de famine aiguë, ou les normes de rationnement entre groupes de population et dans le temps. Le propos sur les continuités, d'une borne chronologique à l'autre du projet, mais aussi la description très neuve d'une sortie de guerre peut-être encore plus dure que les années 1930 pour les mineurs du Donbass, en sortiraient renforcés.

Ainsi, l'ouvrage de Tanja Penter s'inscrit dans la lignée des grandes monographies régionales qui, à l'instar du Magnitogorsk de Kotkin ${ }^{5}$, ont considérablement renouvelé notre regard sur l'histoire soviétique.

\section{NOTES}

1. [Les joyeux garçons]. Ces deux films de Grigorij Aleksandrov étaient sortis sur les écrans soviétiques respectivement en 1934 et 1938.

2. Kuromiya Hiroaki, Freedom and Terror in the Donbass. A Ukrainian-Russian Borderland, 1870s-1990s, Cambridge - New York : Cambridge University Press, 1998, p. 283 ff.

3. S'appuyant sur les travaux de Ulrike Goeken-Haidl (Der Weg zurück: Die Repatriierung sowjetischer Zwangsarbeiter während und nach dem Zweiten Weltkrieg, Essen : Klar Text, 2006), Tanja Penter présente une réévaluation, par rapport aux travaux de V. Zemskov, du nombre de rapatriés civils empêchés de rentrer chez eux après la filtration.

4. Donald Filtzer, Soviet Workers and Late Stalinism : Labour and the Restoration of the Stalinist System after World War II, Cambridge - New York : Cambridge University Press, 2002. 
5. Stephen Kotkin, Magnetic Mountain: Stalinism as a Civilization, Berkeley: University of California Press, 1995. 